\title{
Nursing Students in Clinical Placements Learning in Dyads: A Feasibility Study Using a Non-Randomized Pilot Trial
}

Kelley Tousignant

University of Ottawa, ktousign@uottawa.ca

Amanda Vandyk

University of Ottawa, avandyk@uottawa.ca

Michelle Lalonde

University of Ottawa, michelle.lalonde@uottawa.ca

Sophie Bigras

University of Ottawa, sophie.bigras@uottawa.ca

Sarah Roggie

University of Ottawa, sarah.clugston@uottawa.ca

Kerri-Lynn Weeks

Algonquin College of Applied Arts and Technology, weeksk@algonquincollege.com

Michelle Morley

Algonquin College of Applied Arts and Technology, morleym@algonquincollege.com

Jean-Daniel Jacob

University of Ottawa, jeandaniel.jacob@uottawa.ca

Follow this and additional works at: https://qane-afi.casn.ca/journal

\section{Recommended Citation}

Tousignant, Kelley; Vandyk, Amanda; Lalonde, Michelle; Bigras, Sophie; Roggie, Sarah; Weeks, Kerri-Lynn; Morley, Michelle; and Jacob, Jean-Daniel (2021) "Nursing Students in Clinical Placements Learning in Dyads: A Feasibility Study Using a Non-Randomized Pilot Trial," Quality Advancement in Nursing Education - Avancées en formation infirmière: Vol. 7: Iss. 1, Article 3.

DOI: https://doi.org/10.17483/2368-6669.1235

This Article is brought to you for free and open access by Quality Advancement in Nursing Education - Avancées en formation infirmière. It has been accepted for inclusion in Quality Advancement in Nursing Education - Avancées en formation infirmière by an authorized editor of Quality Advancement in Nursing Education - Avancées en formation infirmière. 


\section{Nursing Students in Clinical Placements Learning in Dyads: A Feasibility Study Using a Non-Randomized Pilot Trial}

\section{Cover Page Footnote}

Acknowledgements We acknowledge the support of clinical instructor Sherley Etienne as well as the unit personnel in the implementation of this intervention. We would also like to thank Dr. Paula Forgeron, the Director of the University of Ottawa School of Nursing for her support of the project. Remerciements Nous aimerions souligner le soutien reçu de Sherley Etienne, enseignante clinique, et des membres de l'unité de soins pour la mise en œuvre de cette intervention. Nous tenons aussi à remercier la Dre Paula Forgeron, directrice de l'École des sciences infirmières de l'Université d'Ottawa, pour son soutien. 
Baccalaureate nursing education programs provide students with clinical placements to ensure that they are prepared to successfully achieve professional practice standards upon entry to practice (College of Nurses of Ontario [CNO], 2014). Delivering these hands-on learning experiences has become increasingly difficult within existing health care environments given scientific advances, shifting patient demographics, changes in the nature of practice settings, and limited resources (Canadian Association of Schools of Nursing, 2011; Ironside et al., 2014; Luhanga, 2018). In our own educational institution, we were required to increase clinical instructorstudent ratios from one instructor for six students to one instructor for eight students. This change increased workloads for instructors, decreased time for teaching and evaluating students' practice, created safety concerns, and lowered instructor and student morale. These negative consequences led us to search for potential alternative structures and processes to better manage the increased ratio. We identified formal pairing of nursing students to work collaboratively (i.e., together in dyads) on patient assignments as a viable solution. The approach has been used with second- and third-year nursing students within acute care settings in Canada and the United States, and results indicate that it improves quality and efficiency of clinical instruction, allows for better use of limited clinical resources, builds students' self-confidence, reduces anxiety, and promotes teamwork (Austria et al., 2013; Dorner et al., 2019; Holst \& Horberg, 2013; Ott \& Succheralli, 2015; RuthSahd, 2011; Trueman et al., 2014). Unfortunately, research to date does not include much direction about how to design and implement student pairs in clinical education. As a result, many faculties grapple with how to create and sustain such educational transformations within their particular contexts (Cabaniss, 2014; Del Prato et al., 2011; Ironside \& McNelis, 2011). In this article, we describe the development and feasibility testing of a clinical education intervention in which students work in dyads during their first clinical experience, which takes place in the second semester of second year.

\section{Background}

Clinical exposure is fundamental to nursing education, with about $50 \%$ of student learning occurring in clinical practice settings (Council of Ontario Universities [COU], 2013). Clinical education allows students to safely practise their nursing skills within a professional milieu (Cooper et al., 2015; Levett-Jones et al., 2015). With recent changes to our health care system and an increase in the number (and size) of professional programs, we are amid a "clinical education crisis" (COU, 2013, p.10.), which is resulting in shortages of placements, preceptor burnout, increased costs, fewer patients available for student practice, and fewer and less experienced nurses acting as mentors (COU, 2013; Ott \& Succheralli, 2015; Ruth-Sahd, 2011). Educators must acclimate to this new context and continue to adequately prepare their graduates for safe, quality patient care (Luhanga, 2018; Ott \& Succheralli, 2015). Adjusting to the new clinical landscape is difficult, and clinical instructors struggle to spend individual time with students to provide appropriate guidance, supervision, and feedback (Ironside \& McNelis, 2010).

Nursing students also face many challenges and stressors when completing their clinical placements because they view this milestone as a critical juncture in their educational and professional journey (Cooper et al., 2015). Transitioning from university classes to clinical practice, nursing students are confronted with experiences that affect them both personally and professionally as they attempt to translate theoretical knowledge into the clinical environment (Cooper et al., 2015). Nursing students describe feeling unprepared, nervous, anxious, and worried when starting their placements and have expressed concerns about bullying, practical difficulties, patient safety, mistakes, and work outside the scope of their practice (Levett-Jones et al., 2015). Levett-Jones and 
colleagues (2015) noted that although curricula are designed to adequately prepare students for their clinical placements, student perceptions and experiences are markedly different.

\section{Traditional Placements}

According to Luhanga (2018), the traditional faculty-supervised clinical education model used in most Canadian undergraduate nursing programs involves a registered nurse, employed by an academic institution, as the clinical instructor who supervises nursing students as they practise nursing care within a health care setting. The clinical instructor is responsible for overseeing the teaching and learning processes, developing patient assignments, conducting student evaluations, and acting as a role model and coach. While this model requires direct supervision of students by a qualified clinical instructor, high student-to-instructor ratios mean that it is not always possible to guarantee the level of supervision, support, and attention needed by most novice students to ensure their success. Yet clinical instructors have an ethical and a professional obligation to ensure that nursing graduates are adequately prepared with the skills required to deliver safe, quality patient care (Luhanga, 2018). This tension results in clinical instructors who feel inadequate in their role (Ironside \& McNelis, 2010) because they spend much of their time coaching students through technical tasks (e.g., medication administration), which limits their ability to effectively engage students in holistic care, critical thinking, and decision making (Ironside et al., 2014; Luhanga, 2018).

\section{Clinical Education Using Student Dyads}

Dyadic learning is a student-centred pedagogy that facilitates the transfer of nursing knowledge between peer learners (Trueman et al., 2014). It requires students to work collaboratively and support each other as they develop skills and knowledge related to their professional role (Carey et al., 2018). In the nursing educational context, a dyad is defined as two nursing students who are in the same clinical rotation-with the same clinical instructor-who share the same patient assignment (Ott \& Succheralli, 2015). Through this approach, the two students and the clinical instructor form a team, which exposes students to the interdependency of teamwork and delegation in a safe, structured manner (Carey et al., 2018; Dorner et al., 2019; Ott \& Succheralli, 2015). According to Ruth-Sahd (2011), student dyads create an educational community that improves learning, patient outcomes, and teamwork. Even when peer learning occurs informally, incidentally, or in an ad hoc fashion, it helps to mitigate student challenges, reduce anxiety, and enhance knowledge, competence, and confidence (Carey et al., 2018; Ott \& Succheralli, 2015). Of note, collaborative learning requires a supportive environment (Holst \& Horberg, 2013; Trueman et al., 2014), and thus it is preferable to implement this approach formally in a collegial and safe setting.

Proponents of the dyad approach to clinical learning suggest that paired students develop a sense of "us" that promotes security, trust, and independence (Holst \& Horberg, 2013). In a recent systematic review representing five countries and four health-related disciplines, Carey and colleagues (2018) identified three key benefits to learning in pairs, as well as challenges to consider. While the benefits are similar to those presented above, challenges were related to (1) being able to accurately define the roles of the individual students composing the pair and (2) ensuring that learning experiences are equally shared between the paired students.

Pairing students for their clinical placements is a viable strategy for undergraduate nursing students, and the benefits appear to outweigh the challenges. Furthermore, this model is operationalizable within current academic and professional landscapes because it allows for a higher student-to-clinical instructor ratio while decreasing the number of patients for whom the clinical 
instructor shares responsibility. Yet evidence informing the design, implementation, and testing of the approach is limited (or arguably non-existent). Therefore, the purpose of this study was to develop a protocol for a clinical education intervention using dyads and to assess the feasibility of implementing the approach with second-year nursing students in their first clinical placement. The objectives were (1) to evaluate and refine data collection procedures and outcome measures, (2) to evaluate the acceptability and sustainability of the intervention, and (3) to identify weaknesses of the intervention or threats to future implementation.

\section{Methods}

\section{Design}

This was a feasibility study as articulated by Orsmond and Cohn (2015). According to these authors, "feasibility studies focus on process and are designed to answer the question, 'Can it work?' and begin to evaluate whether the intervention shows promise. Feasibility studies are the initial phase of developing an intervention. Conducting a feasibility study is a developmental learning process in which the study procedures and intervention can be adapted as necessary during the study to achieve the most promising outcomes" (p. 6).

\section{Setting}

The bachelor of science in nursing at our university located in central Canada is a four-year undergraduate degree program. It is offered jointly with two colleges in both official languages and is mandated to prepare generalist nurses. Students who complete the bachelor of science in nursing are eligible to write the licensure exam to become a registered nurse. The program also leads to graduate studies (master's, nurse practitioner, and doctorate) in nursing sciences. All students are required to complete eight clinical placements as part of their four-year degree program.

\section{Usual Clinical Placement Structure}

The usual first clinical placement consists of a five-week rotation on either a transitional care or rehabilitation unit in one of several hospitals in the city. Transitional units generally provide medical care to a primarily senior population with multiple comorbidities who are waiting placement in long-term care. Rehabilitation units generally provide restorative care to increase patient functionality and subsequent reintegration into the community. In a traditional clinical placement, one clinical instructor is responsible for supervising and evaluating students. Clinical shifts last eight hours and are completed twice a week. Each student receives an individual patient assignment consisting of one to two patients and are accountable for the full care. Assignments (development of a personal learning objective, a care plan, three reflective notes, and a health promotion plan) are submitted individually, and each student is evaluated separately using the existing evaluation framework (Bourbonnais et al., 2008). The clinical placement is assigned a course coordinator who is responsible for overseeing the placement, which includes, for example, mediating a learning plan when a student is not meeting one or more course objectives, monitoring the appropriateness of the teaching-learning environment, and liaising with the agencies.

\section{Intervention}

\section{Guiding Principles}

Before developing the intervention protocol, we established guiding principles to direct our dyad approach to clinical education. These guiding principles were informed by existing evidence 
(Austria et al., 2013; Carey et al., 2018; Dorner et al., 2019; Palsson et al., 2017), as well as consultation with experts at our institution in clinical education:

1. Minimizing the number of patients per clinical instructor while requiring no additional human or financial resources

2. Randomly assigning student dyads

3. Maintaining the dyads throughout the clinical placement

4. Treating the paired students as a unit and having them complete all nursing care and skills together

5. Requiring the paired students to collectively present questions and problems to the clinical instructor after brainstorming solutions together

6. Requiring each student to independently maintain a clinical worksheet and be prepared for all aspects of patient care

7. Requiring each student to independently complete the academic assignments related to the clinical placement

8. Evaluating students separately and tracking skill acquisition independently

\section{Intervention Protocol}

To maintain the integrity of the existing clinical placement and the associated course objectives, we devised a dyad-pairing structure consisting of two options. This pairing structure aimed to ensure students received an equitable assignment and were equally able to engage in performing and observing nursing care, in accordance with the course objectives and year-two scope of practice. Specifically, students were to be designated as Student1 or Student2 and assigned to two care responsibility options, which were meant to alternate between the paired students each shift. Option A included assessment, vital signs, shift report, and all associated documentation. Option B included medications, treatments, and all associated documentation. Jointly, the paired students were responsible for personal care and lifts and transfers. Although each student would be assigned to certain nursing interventions, all aspects of care were to be provided by the dyad. Therefore, when Student1 performed a nursing intervention, Student 2 was supposed to participate as an observer and provide mentoring, feedback, and support. The CNO's Standards of Practice, agency guidelines, and university requirements were adhered to, thereby ensuring that whomever provided the assessments, treatments, or medications was also responsible for the documentation of said intervention (CNO, 2008). This process permitted the division of responsibilities while ensuring safety and accountability standards.

Each week, students' patient assignments and pairing structure were to be posted at the agency by the clinical instructor using a dyad clinical assignment sheet. The intent was for students to independently research their patient assignment and complete the dyad clinical care map and worksheet in advance of assuming their patient assignment. The intervention protocol was developed to guide the five-week clinical placement.

\section{Procedures}

To ensure an even number of students in each clinical placement, we increased clinical group sizes to eight students. The intervention proceeded as follows: Week1, Day1 students were randomly assigned to dyads, completed the pre-intervention questionnaires, and participated in an orientation to the unit. Week1, Day2 proceeded as normal with the dyads completing a buddy shift with a registered nurse working on the unit. Week2 Day1 students received their first patient assignment. During Week2 to Week5, students completed their clinical rotation as usual following 
the pairing structure described above. As per the course objectives, during Week2 and Week3, each dyad received a one-patient assignment, which was subsequently increased to two patients during Week4 and Week5. Students' clinical skill acquisition was tracked by the instructor on a dyad skills checklist throughout the five weeks, and students completed the post-intervention questionnaires at the end of the clinical rotation. Each of the documents described above are available on request from the authors.

\section{Trouble-Shooting Algorithms}

The dyad approach necessitates that students share in learning and clinical experiences, and thus we anticipated that missed opportunities might occur when there was a lack of preparation and direction, differences in personalities, absences, and mismatched learning styles (Carey et al., 2018; Ott \& Succheralli, 2015). To mitigate these potential problems, we created dyad algorithms to guide intervention-related decisions when (1) a group had an odd number of students because of student withdrawal or failure (Figure 1); (2) students were late or required short- or long-term absences (Figure 2); (3) one of the paired students was unprepared (Figure 3); (4) one student was not meeting one or more of the course objectives and required a learning contract (Figure 4); and (5) conflict occurred between the paired students (Figure 5). The potential problems were determined from the literature, in addition to those we routinely encounter in our clinical placements, and the approach to mitigating them proposed in the algorithms was determined through discussion and consensus with clinical education experts at our institution and collaborative college sites.

\section{Instruments and Data Collection}

Instruments included two surveys, an author-developed tool to track evaluation of clinical skills, and a narrative form based on a SWOT (strengths, weaknesses, opportunities, and threats) analysis. The outcomes chosen (described below) provide pivotal information about the viability of the intervention from a practical standpoint, including its effect on student learning and clinical instructor workload.

Surveys were the Nursing Student Self-Efficacy Scale (NSSES) by Stump and colleagues (2012) and the Verbal and Social Caring Interactions questionnaire (VSI-NS) by Rask and colleagues (2018). We included items on these surveys that addressed their usefulness and acceptability (i.e., Was the survey easy to complete? Did you understand all of the questions? Were the questions relevant to your learning? Are there questions related to your communication or skills missing from this survey?). To evaluate and refine data collection procedures and outcome measures, students completed the NSSES and VSI-NS surveys before and after the intervention.

The NSSES is a 26-item self-report questionnaire about nursing student self-efficacy. Using 5 -point Likert scales $(0=$ not confident at all to $5=$ completely confident $)$ nursing students rate their confidence in their ability to perform a series of nursing skills. The tool was developed using the item response theory, and all items demonstrated an acceptable model fit using the chi-square test with an alpha of .001, with a review of the item functions showing that $40 \%$ of the items provided high and 20\% moderate information about self-efficacy (Stump et al., 2012). With permission from Dr. Stump, we modified the skills included in the instrument to appropriately reflect the course objectives and the second-year student's scope of practice.

The VSI-NS was used to measure students' verbal, social, and interactional skills (Rask et al., 2018). This self-report instrument includes 31 items for which nursing students rate their perceptions (i.e., how important it is, how difficult it is, how capable they are) regarding each 
statement using an ordinal scale (not at all, to some degree, high degree, very high degree). The VSI-NS questionnaire has an adequate internal consistency with a Cronbach's alpha equal to 0.93 for the entire questionnaire (2018). Rask and colleagues completed a factor analysis and reported the items loaded on four factors: "inviting to talk about feelings and thoughts," "building a caring relationship," "encouraging social and practical aspects in daily life," and "caring towards health and well-being."

A Year-Two Skills Checklist was developed for use by the clinical instructors to track students' skill acquisition, as well as their availability to supervise and evaluate the required skills. The checklist consists of 15 skills that students complete during the placement. The list of skills is levelled to students' scope of practice and is consistent with what is currently taught and evaluated in the second-year clinical course. The skills include, for example, assessment, mobility, personal care, isolation precautions, sterile technique, medication administration, and documentation. The Year-Two Skills Checklist was used each clinical shift throughout the intervention.

To assess the acceptability, sustainability, and limitations of the intervention, we collected weekly reflective summaries from the clinical instructors assigned to the dyad groups. These reflections were narrative SWOT analyses completed using a form that we created for this purpose. For each narrative reflection, we asked the clinical instructors to reflect on the effect of the intervention on students, staff, patients, and them as educators. Halfway through the study, we modified the form to include a checklist of commonly identified strengths and weaknesses to decrease the burden on the clinical instructors. Further, we had several informal conversations with the unit managers and educators, other clinical instructors, and nurses working on the units about all aspects of the intervention, including the pairing structure, care options, and trouble-shooting algorithms. These conversations occurred ad hoc, and we took detailed notes afterwards. All stakeholders were aware that we were taking notes and consented to this verbally.

\section{Data Analysis}

We analyzed the completed surveys to determine their suitability as data collection instruments and outcome measures. Specifically, we verified the surveys for completeness, identified student comments regarding the usefulness of the measure or burden of completion, assessed the accuracy and relevance of the answers to the demographic questions, and tallied scores to determine whether the responses provided were congruent with our expectations. The frequencies with which skills were performed under the direct supervision of the clinical instructor were tallied, and we identified the skills most and least often completed by students.

Using a simple content analysis approach (Graneheim \& Lundman, 2017), we analyzed the data obtained through the narrative reflections to identify patterns within the reported strengths, weaknesses, opportunities, and threats. The same process was used to analyze data obtained through informal feedback from stakeholders and to assess the usefulness of the problem-solving algorithms.

\section{Rigour and Ethics}

We followed the feasibility study approach as articulated by Orsmond and Cohn (2015) and the Consolidated Standards for Reporting Trials (CONSORT) extension to randomized pilot and feasibility trials guided reporting (Eldridge et al., 2016). Ethics approval was obtained through our institution's research ethics board on 25-06-2019 (REB\# H-05-19-3966). 


\section{Results/Findings}

In total, three clinical groups, consisting of 24 students, and two clinical instructors completed the study. Two groups were part of our second-year English program cohort, and one group was from our second-year French program cohort. Twenty SWOT analyses were submitted by the instructors, and 16 skills checklists were completed. Approximately five informal conversations about the intervention protocol and procedures occurred with students and unit managers.

\section{Data Collection and Outcome Measures}

Overall, the NSSES was completed more often and with greater ease than the VSI-NS. Students indicated that the VSI-NS was difficult to finish and too long, with some of the items seen as ambiguous or not answerable based on their experiences. The demographic questions were answered appropriately; however, we identified a need to include one additional question ("Have you completed a previous clinical placement?"). In all cases, the responses to the instruments were congruent with our expectations and student scores shifted in the anticipated direction. Based on feedback from the instructors, completing the questionnaires immediately following the last clinical shift was feasible. Given that the NSSES was relevant, understandable, and simple to complete, we will retain this tool for future full implementation of the intervention.

\section{Acceptability and Sustainability}

The intervention was deemed acceptable by the clinical instructors, as well as the managers and educators, of the units. We received no negative feedback regarding the intervention or the workload required to implement the intervention properly. From a sustainability perspective, the intervention allowed for the safe implementation of larger group sizes without negatively affecting the learning environment or the integrity of the course objectives.

\section{Supervision of Clinical Skills}

Clinical instructors directly observed all skills for all students at least once during the clinical placement, with head-to-toe assessments, vital signs, personal care, and medication administrations supervised for all dyads each shift. Skills least often observed included patient ambulation and feeding.

\section{Problem-Solving Algorithms}

Our instructors encountered students who had difficulties meeting the clinical learning outcomes, requiring instructor intervention of learning contracts. In all cases, these struggling students represented one student within a dyad; there were no cases of paired students both exhibiting difficulties. Weaknesses were evident in the domains related to skill acquisition, organization, teamwork, confidence, communication, and professionalism. The clinical instructors reported that the problem-solving algorithms helped them navigate issues related to lateness, absences, unmet course objectives, and unprepared students. The instructors suggested some points of clarification to the algorithms, which included adding language to reinforce student privacy when implementing a learning contract and allowing more flexibility when students return to clinical following an absence.

\section{Strengths and Weaknesses for Clinical Instructors}

Several strengths and a few important weaknesses were evident in the clinical instructors' narrative reflections related to their role. Strengths of the intervention pertained to their capacity to 
teach and interact meaningfully with students. For example, when compared to their past teaching experiences using traditional placements, the clinical instructors reported being able to develop better rapport with their students and to spend more time teaching nursing care: "I'm teaching more than I have in the past, where my role was more coordination, and I relied heavily on the nursing staff to teach." The clinical instructors also reported having more opportunity to directly observe their students' practice: "[I was] able to observe each student perform a head to toe assessment and complete medications ... I have never been able to do that so thoroughly." Additional strengths of the dyad approach included having fewer patient assignments to track (and thus better knowledge of each patient and their care requirements), as well as benefits related to peer support on the nursing care provided: "Student conversations resulted in ideas for interventions and reassurance to try them ... this leads to better nursing care."

Weaknesses of the intervention related to the clinical instructor role included trouble reconciling the different learning needs of the paired students, as well as ensuring that students did not divide their workload: "I am able to see their differing levels of preparation through their worksheets and care maps ... I see them as individuals, not simply as dyads," and "As students get busier their temptation is to divide and conquer ... they had to be reminded to work in pairs."

\section{Strengths and Weaknesses for Students}

The clinical instructors' narratives included their reflections on the strengths of the intervention for their students. They perceived their students to be less anxious and more confident in their decisions compared to students they taught using a traditional approach. Further, they saw the benefits related to peer teaching, parallel learning, and the sharing of knowledge between the paired students: "Through maintaining the same partners, students were able to discuss answers ... they learn together ... students were able to discuss together the priority diagnoses and how they would intervene, and they understand the expectation level of preparation by comparing themselves to their partner." From a weakness perspective, the clinical instructors reported that by using the dyad approach, there was a risk that less competent students would become dependent on their partner for direction and support.

\section{Strengths and Weaknesses for Patients and Staff Nurses}

The clinical instructors reported that student teamwork resulted in patients' needs being met quicker, which improved patient safety and social stimulation. From a negative perspective, the clinical instructors noticed that some patients felt overwhelmed with the number of people in the room and required reassurance and reminders regarding the role of students. Staff nurses who provided feedback about the intervention indicated that because the clinical instructor was more present and hands-on, they felt less burdened by teaching obligations and were able to use their time more effectively. A manager noted that "students were more visible and engaged on the unit." Conversely, staff nurses who do not want to mentor students posed a threat to the intervention because these nurses were much less likely to agree to two students compared to one.

\section{Threats to Future Implementation}

There were no obvious threats to the implementation of the intervention.

\section{Discussion}

Using peer learning in undergraduate nursing programs has shown positive effects; however, less is known about the outcomes of this strategy when used in clinical education. Research to date provides little direction on how to design and implement student dyads in clinical 
placements. Throughout this study, we pilot-tested this approach, exploring the strengths and threats to future implementation, as well as the methods used to evaluate its effectiveness. We also learned the needs and expectations of our stakeholders, who are instrumental in supporting our students' learning. Overall, our results suggest that the intervention was well received and feasible as designed. Based on the feedback garnered, we refined the problem-solving algorithms and modified one outcome measure. Three areas warranting further discussion are what to do when one student within the dyad struggles to meet course objectives, the potential benefits of peer learning on student anxiety, and how learning in dyads may affect future success in traditional clinical placements.

\section{Struggles with Student Performance}

To gain entry into the nursing profession, students must demonstrate both theoretical knowledge and practical competence. Therefore, it is ethically and professionally necessary to assess students' clinical practice during their pre-licensure nursing programs (CNO, 2019; Dorner et al., 2019; Hunt et al., 2012). This principle, which requires individual assessment, applies regardless of the learning modality (i.e., traditional versus dyads). While the dyad approach means learning and practising happen in pairs, assessment of student competency must still occur individually to meet entry-to-practice standards (Dorner et al., 2019). Each of the difficulties encountered by students are known problem areas for students during their clinical placements generally (Tanicala et al., 2011). To help students overcome challenges in these areas, Luhanga and colleagues (2014) suggest the following strategies: (1) communicating and clarifying issues, (2) encouraging self-reflection, (3) documenting specific behaviours and attitudes, (4) developing learning contracts or plans of action for success, and (5) providing remedial interventions. These techniques are embedded within our traditional practicums and were also adhered to for the dyad intervention. Although further testing of the dyad approach is needed, we believe student difficulties encountered were unrelated to their working in pairs. In fact, Ott and Succheralli (2015) report that when weaker students are paired with stronger students, they tend to rise to the challenge and perform better than expected.

Recognizing the potential challenge of having one student who is not meeting the objectives within the dyads, we designed algorithms to inform how clinical instructors should proceed in these situations. Two students demonstrated difficulties, with one student receiving a learning contract. The clinical instructor assigned to these students followed the provided algorithms and reported that they were straightforward and helpful for her decision making. Having standardized processes in place to navigate known issues in clinical teaching is likely useful for all types of practicums, especially when part-time and contract employees compose the majority of educators working in the clinical domain (Luhanga, 2018; O'Rae et al., 2017).

\section{Impacts of Peer Learning on Student Anxiety}

According to the clinical instructors, students working in dyads appeared to be less anxious than second-year students working in traditional ways. Worry, self-doubt, and performance-related fear are common sentiments experienced by nursing students during their practical learning (Sun et al., 2016), which is more pronounced with the first clinical exposures (Ruth-Sahd, 2011). When not managed, stress and anxiety can present a significant threat to student success in their clinical placements (Moscaritolo, 2009). Instituting innovative curricular changes has successfully reduced student anxiety in medical practicums (Slavin et al., 2014). From a nursing perspective, the use of dyads in clinical education has also been shown to reduce student anxiety (Austria et al., 2013; Carey et al., 2018; Holst \& Horberg, 2013; Ruth-Sahd, 2011). Being able to rely on your peers to 
brainstorm ideas and come to solutions instills confidence in one's actions (Carey et al., 2018; Trueman et al., 2014). By implementing the dyad approach in the first clinical placement, we hope to foster these skills early in our students' academic trajectories and decrease placement-related stress and anxiety in our nursing program.

\section{Students Moving to Traditional Placements}

Reflecting on this project, our team continually discussed the effects the dyad approach may have on student success in future traditional placements. Despite our reading on the topic and the feedback received from our participants that suggests dyads foster student confidence and autonomy by nurturing teamwork and collaboration when deciding upon and implementing nursing interventions (Carey et al., 2018; Trueman et al., 2014), we are mindful of the potential challenges for students as they progress. For example, it is possible that students will experience an increase in their anxiety once they are required to make decisions and provide nursing care alone. Further, it is possible that within the dyads, more competent students masked the shortcomings of their peers. Commonly, within nursing education, students are expected to build upon previously acquired knowledge, and thus we are hopeful that the problem-solving and critical thinking skills garnered during their dyad placements will persist. To better understand the effects of using a dyad approach in the first clinical placement, we intend to follow students from this study into their next clinical placements to identify areas of strength and weakness that may stem from their participation.

\section{Limitations}

There are two limitations to bear in mind when considering the results of this study. First, given the focus on feasibility, we used a small-scale non-randomized design without a comparison group. While this approach allowed us to determine the appropriateness of our intervention, as well as the implementation and evaluation procedures, we had a small sample size and it is important to highlight that we did not evaluate the effectiveness of the intervention. Second, although we collected data from several stakeholders, we did not elicit formal feedback from students and instead relied on the instructors' perceptions of students' success. It is possible that students' perspectives differ from those articulated by our participants. Moving forward, we plan to implement this intervention across all clinical groups in second year and use our college partner as a comparison site (both campuses deliver the same undergraduate program). With this, we will collect both performance indicators from students, as well as qualitative information regarding their experiences of learning in dyads.

\section{Conclusion}

In this paper, we present an intervention protocol for dyad learning during nursing clinical placements, as well as an assessment of its feasibility in terms of implementation and evaluation. The findings of our study support the use of the intervention, with all stakeholder groups (clinical instructors, students, unit managers, and staff nurses) expressing satisfaction with the procedures. Innovative approaches to the delivery of clinical education are needed to ensure appropriate preparation for students within the existing fiscally constrained academic context. Using the dyad structure, clinical group sizes can increase without further burdening clinical instructors. Specifically, their overall patient responsibility is less, with the student-to-patient ratio decreased by half compared to the traditional approach. This has the potential to save money and redirect resources for improved nursing education in other areas. Further, when students work in dyads, there is a built-in safety net because they jointly problem solve and provide nursing care. Fostering a supportive and reciprocal relationship between the paired students can lead to earlier identification 
of errors and improved critical thinking when the observing student is actively engaged. While we consider the results of our study to be promising, further research is needed to determine the effectiveness of this intervention compared to traditional placements. 


\section{References}

Austria, M. J., Baraki, K., \& Doig, A. K. (2013). Collaborative learning using nursing student dyads in the clinical setting. International Journal of Nursing Education Scholarship, 10(1), 18. https://doi.org/10.1515/ijnes-2012-0026

Bourbonnais F. F., Langford S., \& Giannantonio L. (2008). Development of a clinical evaluation tool for baccalaureate nursing students. Nurse Education Practice, 8, 62-71. https://doi.org/10.1016/j.nepr.2007.06.005

Cabaniss, R. (2014). Using transformative learning theory to impact patient care. Journal of Nursing Care, 4(1), 1-4. https://doi.org/10.4172/2167-1168.1000223s

Canadian Association of Schools of Nursing. (2011). Position statement: Education of registered nurses in Canada. https://casn.ca/wp-content/uploads/2014/10/EducationofRNsinCanada Eng.pdf

Carey, M. C., Kent, B., \& Latour, J. M. (2018). Experiences of undergraduate nursing students in peer assisted learning in clinical practice: A qualitative systematic review. JBI Database of Systematic Reviews and Implementation Reports, 16(5), 1190-1219. https://doi.org/10.11124/JBISRIR-2016-003295

College of Nurses of Ontario. (2014). Entry-to-practice competencies for registered nurses. https://www.cno.org/globalassets/docs/reg/41037_entrytopracitic_final.pdf

College of Nurses of Ontario. (2008). Practice Standard: Documentation (Revised 2008). https://www.cno.org/globalassets/docs/prac/41001_documentation.pdf

Cooper, J., Courtney-Pratt, H., \& Fitzgerald, M. (2015). Key influences identified by first year undergraduate nursing students as impacting on the quality of the placement: A qualitative study. Nurse Education Today, 35, 1004-1008. https://doi.org/10.1016/j.nedt.2015.03.009

Council of Ontario Universities. (2013). Integrating clinical education into Ontario's changing health care system. https://cou.ca/reports/integrating-clinical-education-into-ontarioschanging-health-care-system/

Del Prato, D., Banker, E., Grust, P., \& Joseph, J. (2011). Transforming nursing education: A review of stressors and strategies to support students' professional socialization. Advanced Medical Education Practice, 2, 109-116. https://doi.org/10.2147/AMEP.S18359

Dorner, S., Fowler, T., Montano, M., Janisse, R., Lowe, M., \& Rowland, P. (2019). Implementing a peer-learning approach for the clinical education of respiratory therapy students.

Canadian Journal of Respiratory Therapy, 55, 21-27. https://doi.org/10.29390/cjrt-2018$\underline{022}$

Eldridge, S. M., Chan, C. L., Campbell, M. J., Bond, C. M., Hopewell, S., Thabane, L., \& Lancaster, G. A. (2016). CONSORT 2010 statement: Extension to randomized pilot and feasibility studies. British Medical Journal, 355, 1-29. https://doi.org/10.1136/bmj.i5239

Graneheim, U. H., \& Lundman, B. (2017). Methodological challenges in qualitative content analysis: A discussion paper. Nursing Education Today, 56, 29-34. https://doi.org/10.1016/j.nedt.2017.06.002 
Holst, H., \& Horberg, U. (2013). Students learning in clinical practice, supervised in pairs of students-A phenomenological study. Journal of Nursing Education and Practice, 3(8), 113-124. https://doi.org/10.5430/jnep.v3n8p113

Hunt, L. A., Mc Gee, P., Gutteridge, R., \& Hughes, M. (2012). Assessment of student nurses in practice: A comparison of theoretical and practical assessment results in England. Nurse Education Today, 32(4), 351-355. https://doi.org/10.1016/j.nedt.2011.05.010

Ironside, P. M., \& McNelis, A. M. (2010). Clinical education in pre licensure programs: Findings from a national survey. Nursing Education Perspectives, 31(4), 264-265. https://doi.org/10.1016/j.nedt.2020.104522

Ironside, P. M., \& McNelis, A. M. (2011). Transforming clinical education. Journal of Nursing Education, 50(3), 123-124. https://doi.org/10.3928/01484834-20110216-01

Ironside, P. M., McNelis, A. M., \& Ebright, P. (2014). Clinical education in nursing: Rethinking learning in practice settings. Nursing Outlook, 62, 185-191.

http://doi.org/10.1016/j.outlook.2013.12.004

Levett-Jones, T., Pitt, V., Courtney Pratt, H., \& Harbrow, G. (2015). What are the primary concerns of nursing students as they prepare for and contemplate their first clinical placement experience? Nursing Education in Practice, 15, 304-309. https://doi.org/10.1016/j.nepr.2015.03.012

Luhanga, F., Koren, I., Yonge, O., \& Myrick, F. (2014). Strategies for managing unsafe precepted nursing students: A nursing faculty perspective. Journal of Nursing Education and Practice, 4(5), 116-125. https://doi.org/10.5430/jnep.v4n5p116

Luhanga, L. F. (2018). The traditional-faculty supervised teaching model: Nursing faculty and clinical instructors' perspectives. Journal of Nursing Education and Practice, 8(6), 124137. https://doi.org/10.5430/jnep.v8n6p124

Moscaritolo, L. M. (2009). Interventional strategies to decrease nursing student anxiety in the clinical learning environment. Journal of Nursing Education, 48(1), 17-23. http://doi.org/10.3928/01484834-20090101-08

O’Rae, A., Langille, J., Li, A., Sealock, K., \& Rutherford, G. (2017). The evolving role of a clinical instructor in an integrated undergraduate nursing curriculum. Journal of Nursing Education and Practice, 7(4), 87-95. https://doi.org/10.5430/jnep.v7n4p87

Orsmond, G., \& Cohn, E. S. (2015). The distinctive features of a feasibility study: Objectives and guiding questions. Occupational Therapy Journal of Research: Occupation, Participation and Health, 35(3), 1-9. https://doi.org/10.1177/1539449215578649

Ott, L. K., \& Succheralli, L. (2015). Use of student clinical partner dyads as a teaching strategy to facilitate learning. Journal of Nursing Education, 54(3), 169-172. https://doi.org/10.3928/01484834-20150217-13

Palsson, Y., Martensson, G., Leo Swenne, C., Adel, E., \& Engstrom, M. (2017). A peer learning intervention for nursing students in clinical practice education: A quasi-experimental study. Nurse Education Today, 51, 81-87. https://doi.org/10.1016/j.nedt.2017.01.011

Rask, M., Gunilla, A., Jalal, S., Stig, W., Lisbet, A., Monica, C. B., Lise-Lotte, O., Christel, B., \& Gunilla, L. (2018). Validation of the verbal and social interaction questionnaire for nursing 
students-The focus of nursing students in their relationships with patients. Journal of Nursing Education and Practice, 8(4), 81-88. https://doi.org/10.5430/jnep.v8n4p81

Ruth-Sahd, L. A. (2011). Student nurse dyads create a community of learning: Proposing a holistic clinical education theory. Journal of Advanced Nursing, 67(11), 2445-2454. https://doi.org/10.1111/j.1365-2648.2011.05690.x

Slavin, S. J., Schindler, D. L., \& Chibnall, J. T. (2014). Medical student mental health 3.0: Improving student wellness through curricular changes. Academic Medicine, 89(4), 573577. https://doi.org/10.1097/ACM.0000000000000166

Stump, G., Husman, J., \& Brem, S. K. (2012). The nursing student self- efficacy scale. Nursing Research, 61(3), 149-158. https://doi.org/10.1097/NNR.0b013e318253a750

Sun, F. K., Long, A., Tseng, Y., Huang, H. M., You, J. H., \& Chiang, C. Y. (2016). Undergraduate student nurses' lived experiences of anxiety during their first clinical practicum: A phenomenological study, Nurse Education Today, 37, 21-26. https://doi.org/10.1016/j.nedt.2015.11.001

Tanicala, M. L., Scheffer, B. K., \& Roberts, M. S. (2011). Defining pass/fail nursing student clinical behaviors phase I: Moving toward a culture of safety. Nursing Education Perspectives, 32(3), 155-161. https://doi.org/10.5480/1536-5026-32.3.155

Trueman, G., Osuji, J., \& El-Hussein, M. T. (2014). Baccalaureate nursing students' experience of dyadic learning in an acute care setting. Journal of Nursing Education, 53(9), 65-71. https://doi.org/10.3928/01484834-20140821-23 
Figure 1

Intervention-Related Decisions When Unable to Create a Full Set of Dyads

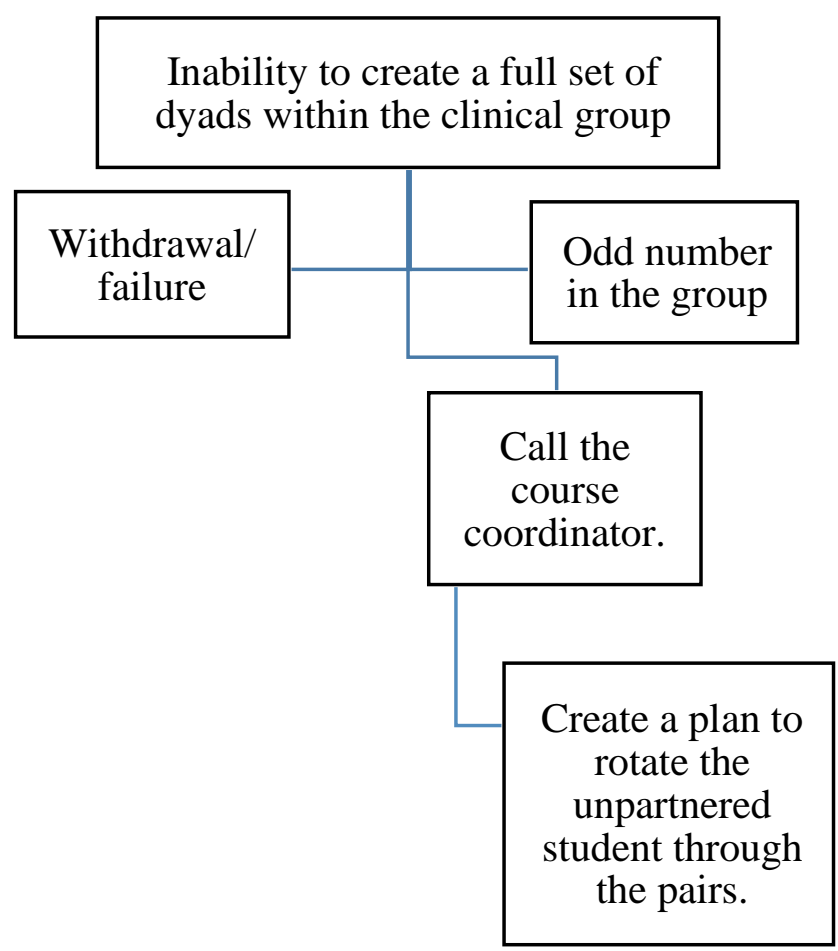




\section{Figure 2}

Intervention-Related Decisions for Absence and Lateness

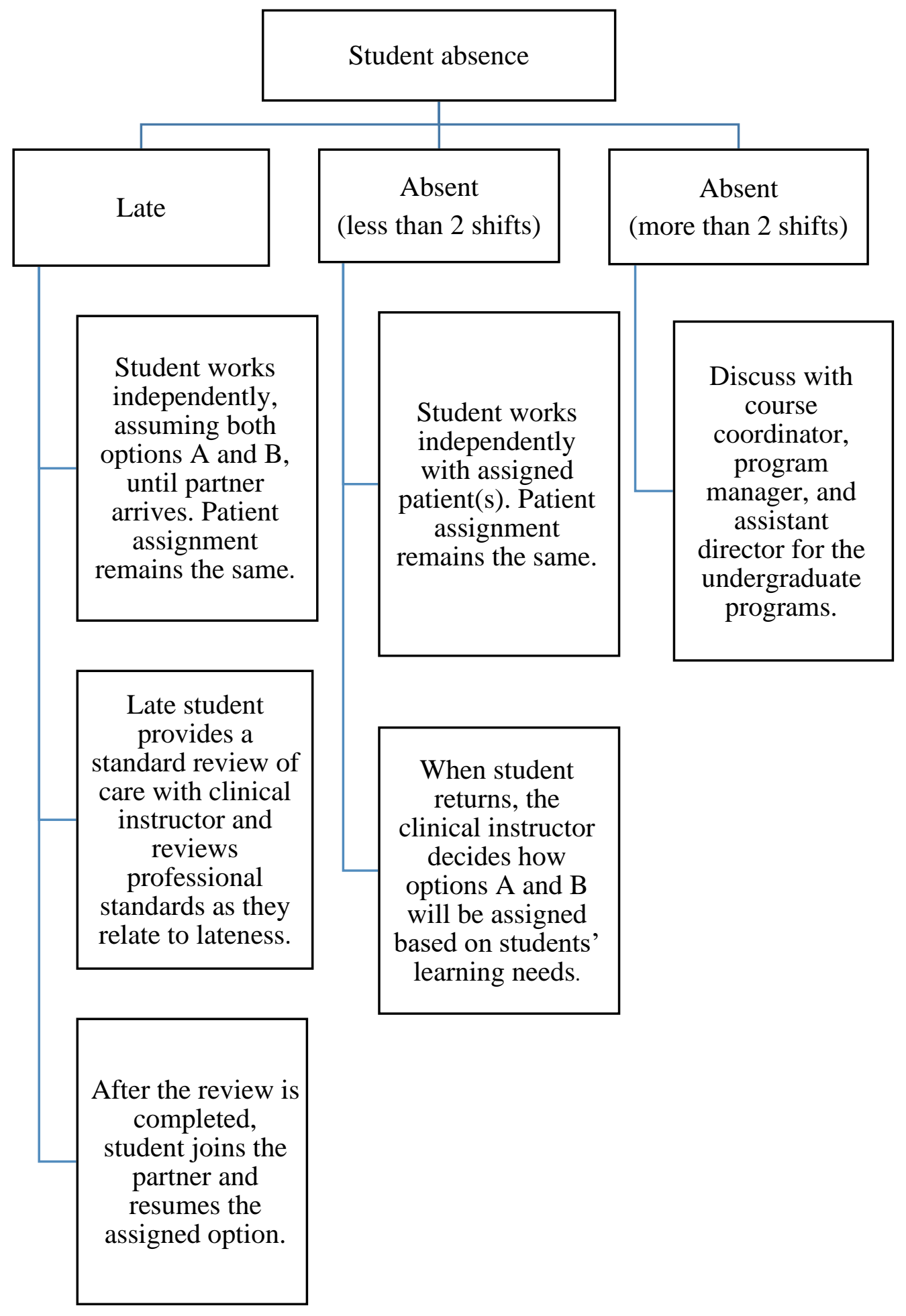




\section{Figure 3}

Intervention-Related Decisions When Preparation Is Lacking

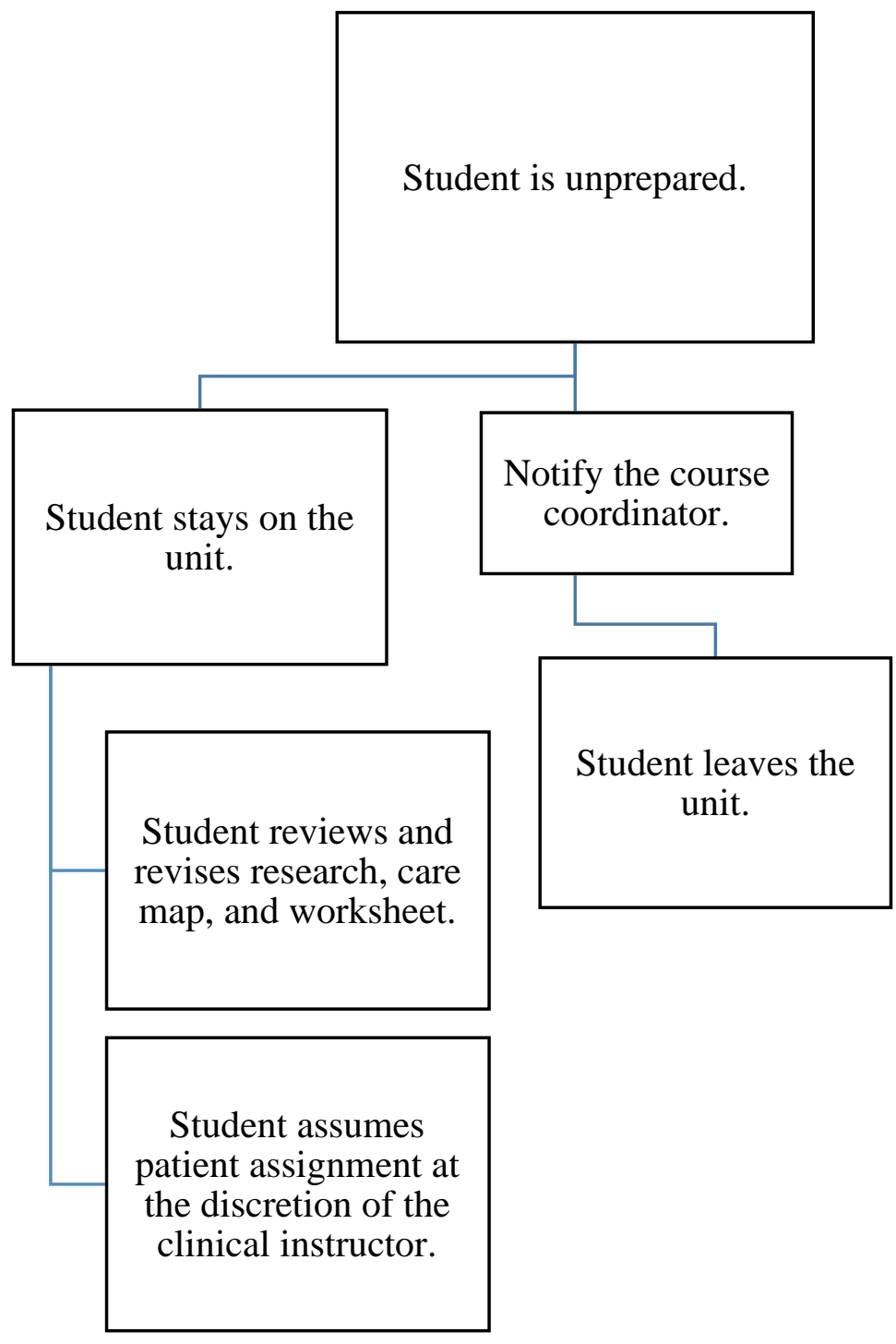




\section{Figure 4}

Intervention-Related Decisions When Objectives Are Not Being Met

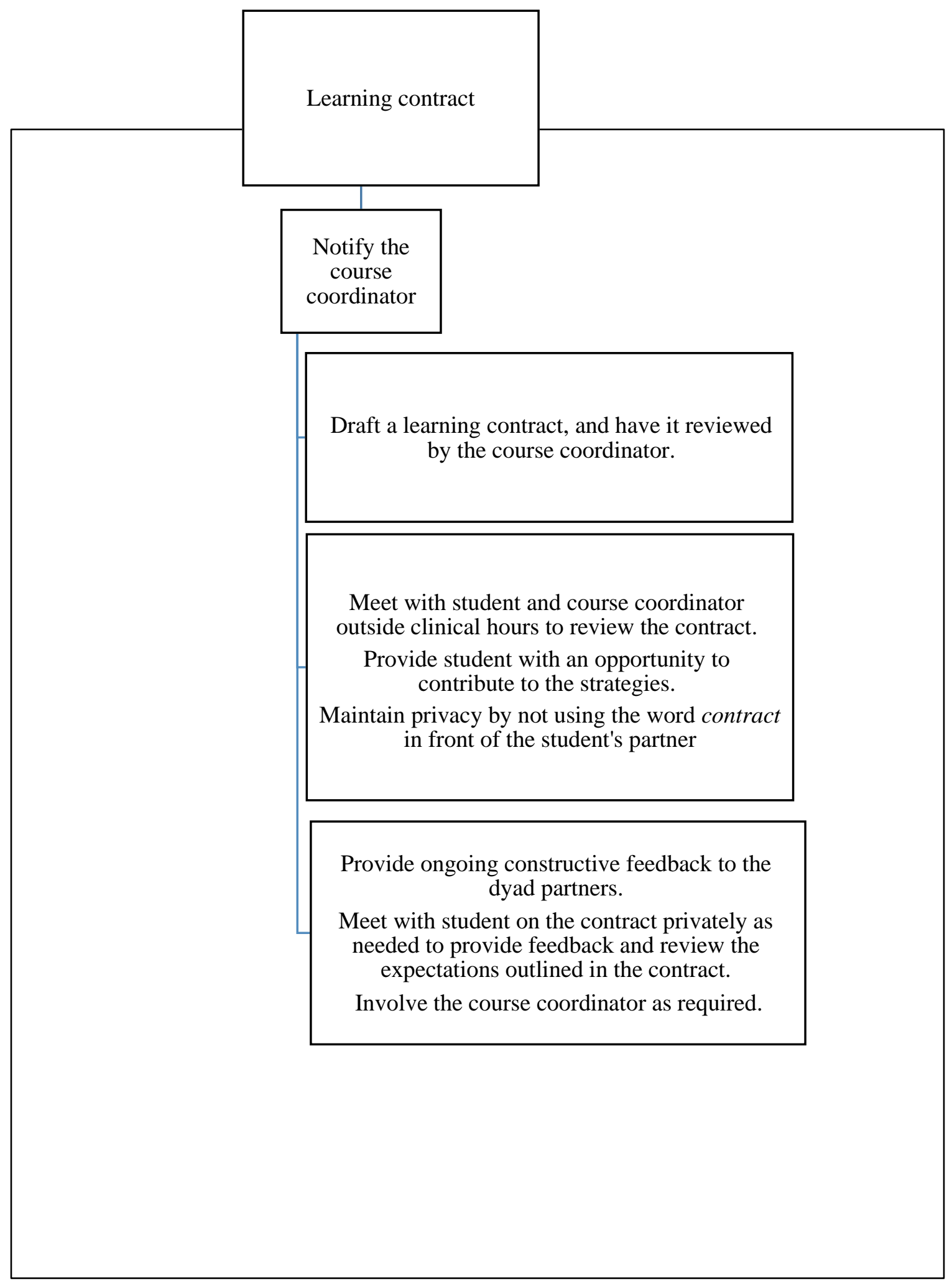




\section{Figure 5}

Intervention-Related Decisions When There Is Conflict within a Dyad

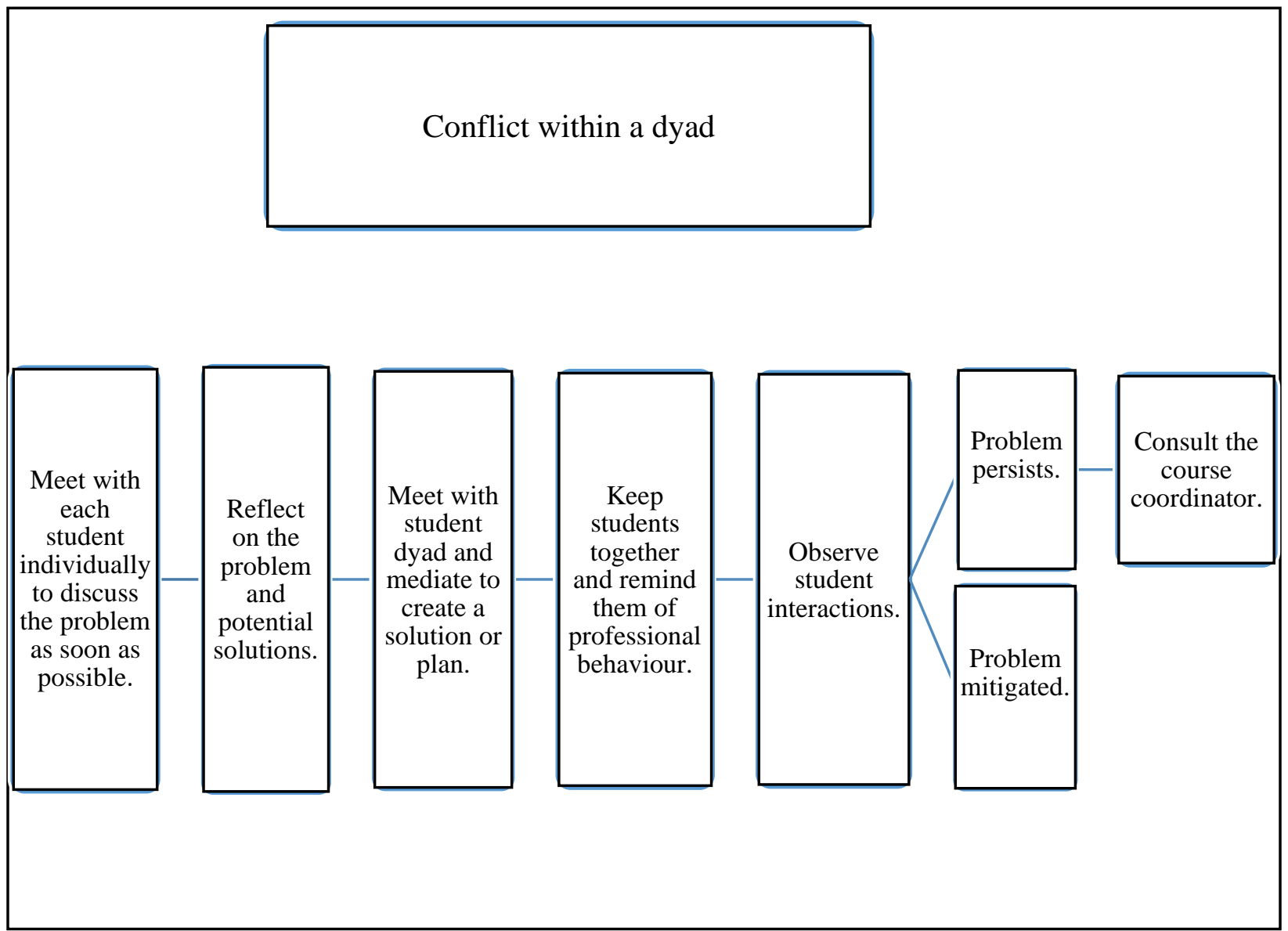

